\title{
English Speech to Sanskrit Speech (ESSS) using Rule based Translation
}

\author{
Pragya Shukla \\ Associate Professor \\ IET, DAVV \\ Khandwa road, Indore (MP)
}

\author{
Akanksha Shukla \\ ME Scholar \\ IET, DAVV \\ Khandwa Road, Indore (MP)
}

\begin{abstract}
The development of such system that automatically recognizes the input speech and translates in another language like Sanskrit is a challenging task. Sanskrit language is much more conjured language. The purpose of this paper is to explain a system which convert the English Speech into English text and then translate that English text into Sanskrit text and again convert that into speech. This system falls into the category of Speech-to-Speech translation. It unifies the isolated words class under the Speech Recognition type, traditional dictionary rule based machine translation approach and text to speech synthesizer. So basically it is classifies into three areas: Speech Recognition, Machine Translation and Speech Synthesis. This system matches tokens [1] from database to differentiate Subject, Object, Verb, Preposition, Adjective, and Adverb. This paper presents approach for translating well-structured English sentences into Sanskrit sentences. Since Sanskrit is free ordering language (or syntax free language) or we can say its meaning won't be change even if the order of words changes.
\end{abstract}

\section{Keywords}

Rule based dictionary approach, Synthesizer, Translator, Speech Recognition, Rule based machine translation

\section{INTRODUCTION}

The Speech is main and convenient mode of Communication among human being. Speech has great efficiency to interact with computers [2]. Speech Recognition is defined as the process of converting spoken words into written text. The goal of speech recognition area is to be developed so it becomes more convenient to interact with the computers. This Speech Recognition module will be used by user; user will give English speech as input. In this we have used isolated class of speech recognition technique. And it will recognize those words only which were stored in file. It means it is a template based approach matching in which whole word matching take place.

The second module is translation. Machine translation [3] is the process of translating one natural language into another language. This process uses the rules and grammatical structure of both the languages to translate source language into target language. Machine translation in Sanskrit is never an easy task because of structural vastness of its grammar but the grammar is well organized and least ambiguous compared to other natural language. The module present concerns with the Machine Translation domain of Natural Language Processing. In the proposed methodology, for translation we decode the meaning of the source input text in its entirety, the translator must interpret and analyze the text, a process that requires deep knowledge of the grammar, semantics, syntax, idioms, etc., of the source language and target language. The translator needs the same in-depth knowledge to re-encode the meaning in the target language. In this we used the dictionary rule based translation approach.

And the last module is synthesizer. Speech Synthesis is the process of generating spoken language for text input. This module concerns the speech synthesizing process. In our system spoken language will generate for Sanskrit text which will produced after the translation process from English to Sanskrit. In this we have record words in our voice and those recordings are stored in database. That is used by system for generating spoken language.

If we compare the syntax of both the languages, English and Sanskrit, English is of the order SVO (subject-verb-object) while Sanskrit is free order language for instance SVO (subject-verb-object) or SOV [4] (subject-object-verb).

For example:-

English text- He will go to school.

Sanskrit text- सः गच्छस्यति विद्यालट्म् (SVO)

Or

सः विद्यालय्म् गच्छस्यति (SOV)

\section{RELATED WORK}

There are various machine translation systems till date. The Desika system developed by Indian Heritage Group, C-DAC, and Bangalore is a NLU system for generation and analysis for plain and accented written Sanskrit texts based on grammar rules of Panini's Asthadhayi rules. The system is also able to analyze Vedic texts. Sanskrit Authoring System (VYASA) project of C-DAC Bangalore is a multipurpose tool for Sanskrit - from being a multi-script editor to a language processing and interpretation tool. It also says that it provides tools for analyses at morphological, syntactic and semantic levels. The Anglabharti [5] employs a pseudo-interlingua approach which analyses English sentences once and creates an intermediate structure called PLIL (Pseudo Lingua for Indian Languages). This is the basic translation process which translates the English source language to. The PLIL structure is further converted to each Indian language through text-generation. Anglabharti-II uses a generalized example base (GEB) for hybridization besides a raw example base (REB). The major components of Anglabharti are:

1. Rule-base: This contains rules for mapping structures of sentences from English to Indian languages. This database of pattern-transformation from English to Indian languages is entrusted the job of making a surface-tree transformations, bypassing the task of getting a deep tree of the sentence to be translated. The approach used in Anglabharti has derived from 
the phrase structure grammar of Chomsky and the c-structure of lexical-functional grammar. The database of structural transformation rules from English to Indian languages forms the heart of the Anglabharti.

2. Sense disambiguator: This module is responsible for picking up the correct sense of each word in the source language. It should be of interest to note that sense disambiguation is done only for the source text. The approach used in Anglabharti may be termed rule-by-rule semantic interpretation. Here, the semantic interpreter is called each time a syntactic rule is applied.

3. Target text generator: Its function is to generate the translated output for the corresponding target languages. They take input of the intermediate form generated by the previous stages of Anglabharti.

4. Multi-lingual dictionary: This contains various details for each word in English, like their syntactic categories, possible senses, keys to disambiguate their senses, corresponding words in target languages.

5. Rule-base acquirer: This prepares the rule-base for the MT system.

Anubharati is a project at IIT Kanpur, dealing with templatebased machine translation from Hindi to English, using a variation of example-based machine translation. Some of the features of Anubharti are based on the emerging trend of third generation of machine translation. The project uses hybrid example-based model for machine translation (HEBMT), combining the strategies used in the pattern/rule-based approach and the example-based approach. MaTra [6] is a project at C-DAC, Mumbai, and has been funded by TDIL. It aims at machine-assisted translation from English into Hindi, essentially based on a transfer approach using a frame-like structured representation. The focus is on the innovative use of man-machine synergy - the user can visually inspect the analysis of the system and provide disambiguation information using an intuitive GUI, allowing the system to produce a single correct translation. The system uses rulebases and heuristics to resolve ambiguities to the extent possible. The Mantra [7] project has been developed by CDAC, Bangalore. The project has been funded by TDIL and later by the Department of Official Languages. Mantra becomes part of Smithsonian Institution's National Museum of American History. The project is based on the TAG formalism from University of Pennsylvania, USA. A sublanguage English-Hindi MT system has been developed for the domain of gazette notifications pertaining to government appointments. In addition to translating the content, the system can also preserve the formatting of input Word documents across the translation. The Mantra approach is general, but the lexicon/grammar has been limited to the sublanguage of the domain. English-Kannada, English-Tamil, English-Assamese Machine Translation systems are also available. ETrans [8] is English-Sanskrit Machine Translator System. By Etrans an English sentence is translated into string of Sanskrit. This system is highly dependent on the database for generating output and to extract the information programming logic have been developed. The software comprised of the following modules:

1. Parse Module

- Input Module

- Sentence Analyzer Module

- Morphological Analysis Module
- $\quad$ Parse Module

- $\quad$ Parse Tree

2. Generator Module

- $\quad$ Mapping

- Output Module

Apart from these big achievements lot of small works all also going in this field of research, like Sanskrit voice engine etc.

Table 1: List of Various Projects on NLP

\begin{tabular}{|l|l|l|}
\hline \multicolumn{1}{|c|}{ Project } & \multicolumn{1}{|c|}{ Language } & \multicolumn{1}{c|}{ Feature } \\
\hline DESIKA & $\begin{array}{l}\text { Analyze Sanskrit } \\
\text { language }\end{array}$ & $\begin{array}{l}\text { Analyze Vedic } \\
\text { text }\end{array}$ \\
\hline ANGLABHARTI & $\begin{array}{l}\text { English to Indian } \\
\text { languages }\end{array}$ & Include corrector \\
\hline ANUBHARATI & Hindi to English & Used HEBMT \\
\hline MATRA & English to Hindi & $\begin{array}{l}\text { Used man- } \\
\text { machine synergy }\end{array}$ \\
\hline ETRANS & $\begin{array}{l}\text { English to } \\
\text { Sanskrit }\end{array}$ & $\begin{array}{l}\text { Used rule based } \\
\text { translation }\end{array}$ \\
\hline
\end{tabular}

In our project we have include Speech recognition and Speech Synthesizing also.

\section{PROPOSED TECHNOLOGY}

Speech Recognition system can be divided into various different classes by describing what of utterance they have the ability to recognize. These classes are classified as following:

1. Isolated words

2. Connected words

3. Continuous Speech

4. Spontaneous speech

We used isolated words class of speech recognition types.

Isolated words: Isolated words [9] usually require each utterance to have quiet (lack of an audio signal) on both side of sample window. It accepts single word and single utterance at a time. These systems listen $\backslash n$ on listen states, where they require the speaker to wait between utterances (usually doing process during the pauses). Isolated utterance might be a better name for this class.

The template based matching approach is used for speech recognition. And whole word matching will take place. In this speech is compared against a set of prerecorded words (templates) in order to find the best match. This has the advantage of using perfectly accurate word models. The engine compares the incoming digital-audio signal against a prerecorded template of the word. This technique takes much less processing than Sub-word matching, but it requires that the user (or someone) prerecord every word that will be recognized - sometimes several hundred thousand words. Whole-word templates also require large amounts of storage (between 50 and 512 bytes per word) and are practical only if the recognition vocabulary is known when the application is developed. For translation generally there are three main approaches used as follows, 
1. Rule based

\section{Statistical based}

Rule Based [10] method for machine translation is divided into another three types,

Rule based translation consists of

1. Process of analyzing input sentence of a source language syntactically and or semantically

2. Process of generating output sentence of a target language based on internal structure each process is controlled by the dictionary and the rules.

The strength of the rule based method is that the information can be obtained through introspection and analysis.

\section{Example Based}

\section{METHODOLOGY}

The main idea behind our system is using isolated technique for Speech recognition by using template based whole word matching technique. And from Speech Recognition process we'll get English text and that English text is translated in target language [11]. The two major tasks for translation are parsing [12] and mapping. The English text is first split into words then the corresponding Sanskrit words to English words looked from the database then using the grammar rules Sanskrit sentence is created.

The overall system in diagrammatic manner is shown below. It consist speech recognition, translation and speech synthesizing.

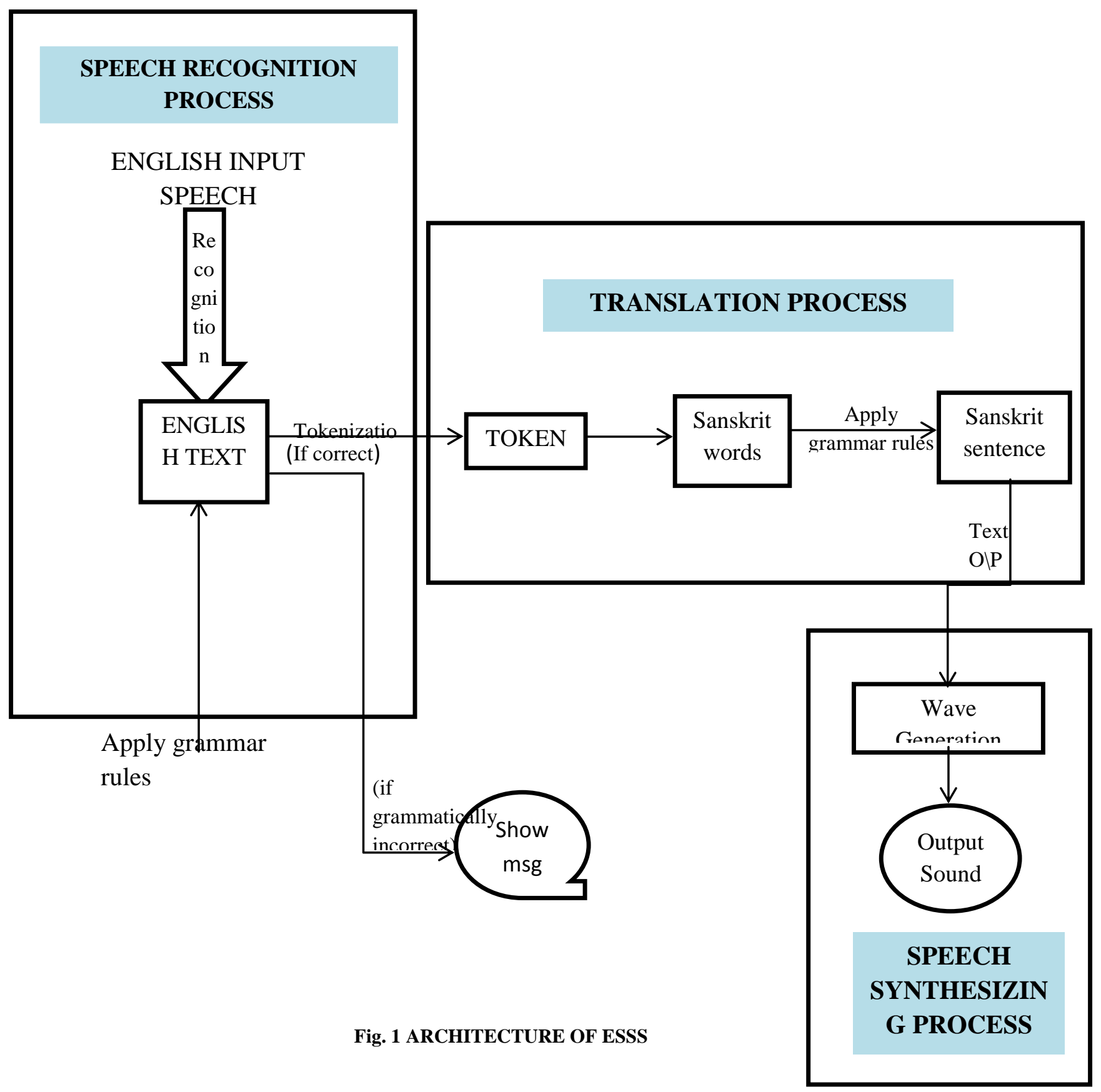


1. Input: In this step the English speech is given as input. Then this speech input is converted into English text. Then this English text is provided for next step.

2. Tokenization Process: In this process the English sentence is split into tokens delimited by spaces, comma, and colon etc. These strings may be composed of simple words. By applying the rules of English grammar assign appropriate category to words like (noun, verb, pronoun etc.)

3. Analysis process: The database developed plays a very important role for analysis process. As it searches the tokens to gather the information. This process takes the tokens as input and gathers grammatical information on that token.

4. Translator process: This module performs the actual translation. The input to this module is the parse. It also interacts with the parser/generator module to get the parse of each word. It then generates appropriate equivalents in English for the morphological details of each word and ultimately presents sentence in the correct order.

5. Parser /generator and mapping: This process checks whether the input sentence is grammatically correct or not. The information gathered from above mentioned process helps in analyzing the grammatical aspect of the sentence and on the basis of the rules the assessment is done. Mapping is done purely on the basis of the information passed from the analysis module. Then it gives the parse of the words, which are used by the sentence former to give the output in a structurally correct sentence.

6. Synthesizing process: When the Sanskrit text is available then it is converted into spoken language. Synthesized speech can be created by concatenating pieces of prerecorded speech that are stored in a database.

Step 1: Give the input as English speech to the system. Then it will recognize those spoken words and will converted them into written text.

For example:

English text- He goes to school.

Step 2: Generate tokens means split sentence into tokens.

As in our case tokens are as follow: He, goes, to, school

Step 3: Analyze the part of speech and related information of each tokens [13].

Like he $=$ pronoun $($ subject $)$

Goes $=$ verb

To $=$ preposition

School =noun $($ object $)$
Step 4: Apply the rules on the tokens. If these tokens follow the rules then it transfer to next step.

For eg. There is rule like

Pronoun+verb+preposition+noun (SVO)

So our example follows this rule. Then forward to next step. Otherwise it will display an error message grammatically incorrect.

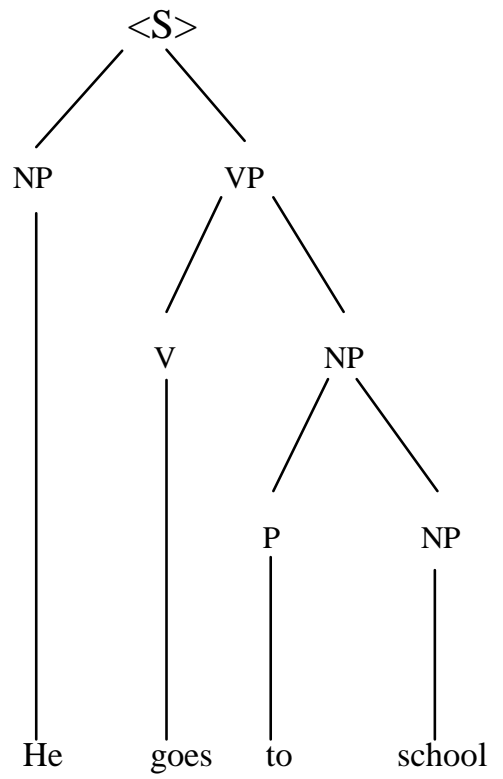

Fig. 2 Parse Tree

Step 5: Then using the information obtained from the above steps generate grammatically correct Sanskrit sentence. Sanskrit text- सः गच्छति विद्यालय्म्

Step 6: Generate the spoken language for written Sanskrit text using speech synthesizer system.

\section{OUTCOME}

The main objective of this system is to develop a translator that translates English into Sanskrit language. This has been done using the rules based translation. When user will enter the English text or can speak then it will convert into Sanskrit sentence. If user wants spoken language for that sentence then he can do that by using speak_sans button. If user enters English text grammatically incorrect then it will display an error message "grammatically incorrect". 


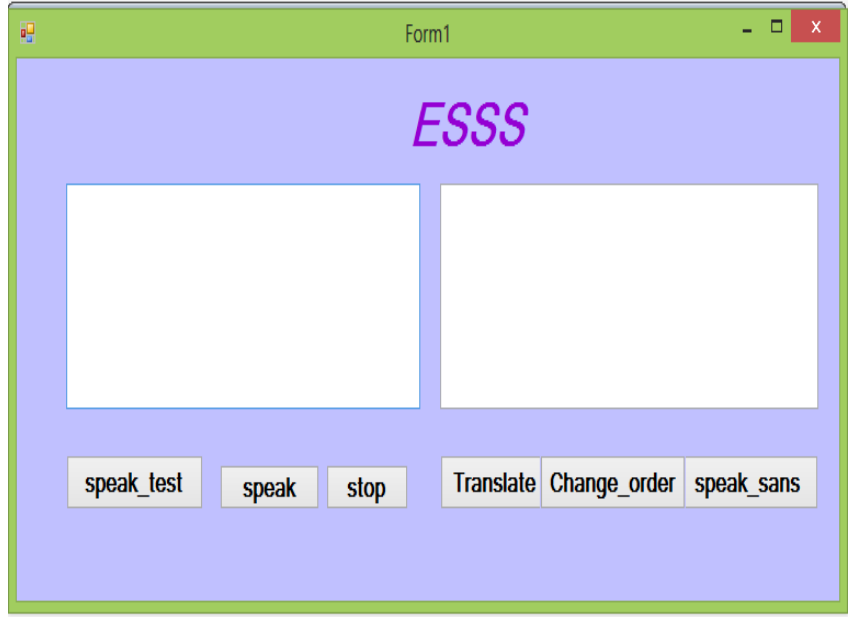

Fig. 3 Snapshot of Output Screen

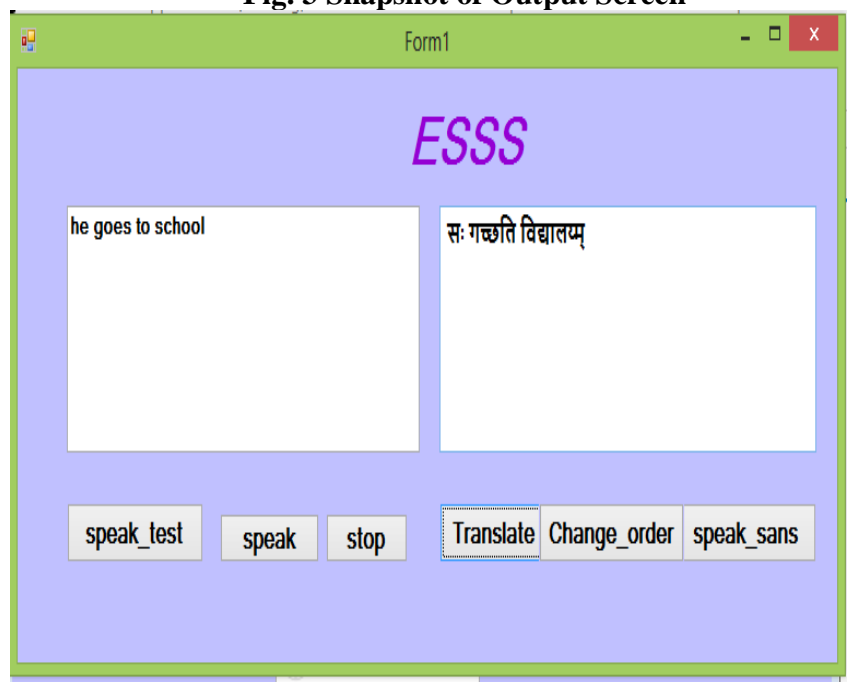

Fig. 4 Snapshot after Sanskrit Translation

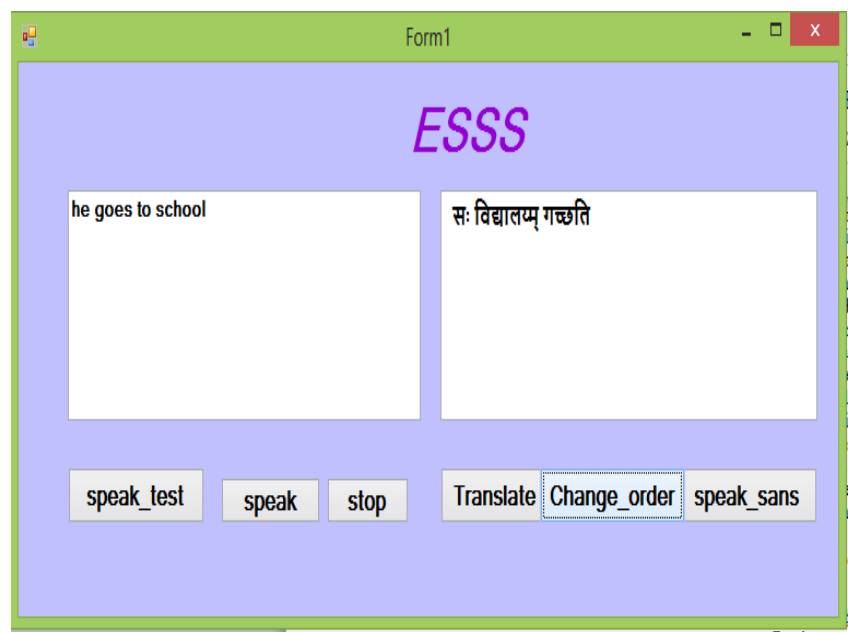

Fig. 5 Snapshot after changing order

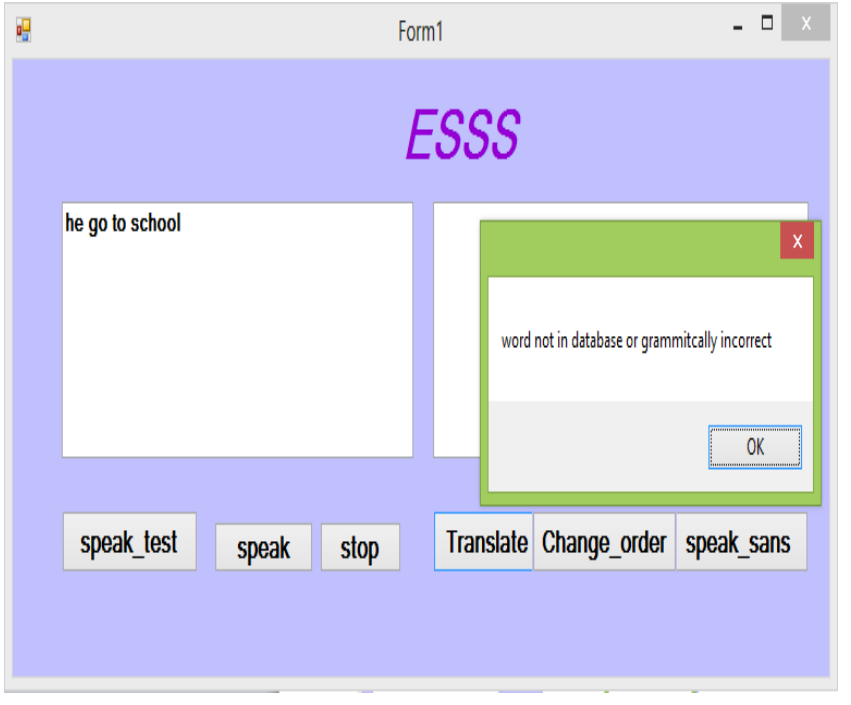

Fig. 6 Snapshot of Grammatically incorrect sentence

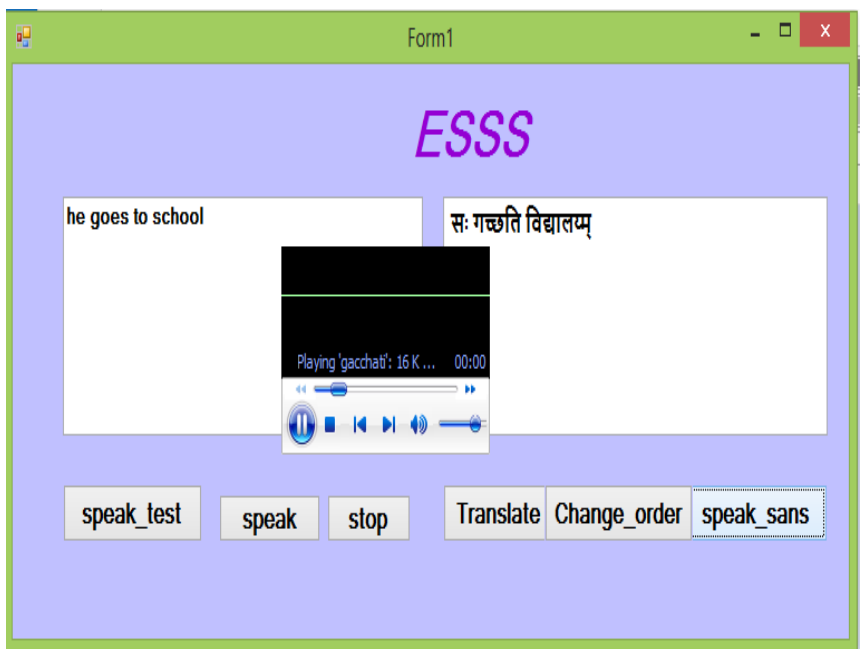

Fig. 7 Snapshot of Synthesizing Sanskrit sentence

\section{CONCLUSION}

The main aim of this paper is to explain a system that converts English speech to Sanskrit speech. It incorporated following process; speech recognition process, translation process and speech synthesizing process. Translation process has been done by rule based translation. The sentence has been first tokenized and then information like parts of speech whether that token is noun, pronoun, adverb, preposition etc. is gathered from the database for the corresponding tokens. For this that token is look in the database and then the Sanskrit word is searched for the corresponding token and the part of speech (POS) information is searched. After looking for all the tokens then system will apply the grammar rules on those tokens and will generate Sanskrit sentence by using information gathered from above. And then pass that Sanskrit sentence to the speech synthesizing process and spoken language is generated. For the synthesizing process also system will have to look up in the database for the recordings of the corresponding Sanskrit words.

This work will be very useful for those students who are learning Sanskrit language. Till now the available translator are just like the dictionaries like word translation. As in our 
system it translates simple sentence not complex sentence. Speech recognition and Speech synthesis has been developed steadily over the last decades and it has been incorporated into several new applications.

\section{REFRENCES}

8. Sarita G. Rathod and Shanta Sondur "English to Sanskrit Translator and Synthesizer (ETSTS)", International Journal of Emerging Technology and Advanced Engineering vol. 2, issue 12, dec. 2012.

9. http://www.ijcaonline.org/archives/volume10/number3/1 462-1976

10. Promila Bahadur, A.K Jain and D.S Chauhan, "English to Sanskrit Machine Translation”, ICWET 2011, Bombay, ACM2011.

11. Rick Briggs, "Knowledge Representation in Sanskrit and Artificial Intelligence", AI Magazine Volume 6 Number 1,1985

12. ANGLABHARTI: a multilingual machine aided translation project on translation from English to Indian languages.

13. http://www.elda.org/en/proj/scalla/SCALLA2001/SCAL LA2001Rao.pdf.
14. http://www.mantra-project.eu/

15. http://thesai.org/Downloads/SpecialIssueNo4/Paper_7EtranS_A_Complete_Framework_for_English_To_Sans krit_Machine_Translation.pdf.

16. Zahi N.Karam,William M.Campbell "A new Kernel for SVM MIIR based Speaker recognition "MIT Lincoln Laboratory, Lexington, MA, USA.

17. U.C. Patkar, P.R. Devale and S.H. Patil, "Transformation of multiple English text to Vocal Sanskrit using Rule Based Technique", International Journal of Computers and Distributed Systems Vol. No.2, Issue 1, December 2012.

18. Pragya Shukla and Akanksha Shukla "A Framework Of Translator From English Speech To Sanskrit Text", IJETAE, vol.3, issue 11, nov. 2013.

19. Vaishali M. Barkade et. al. / (IJCSE) International Journal on Computer Science and Engineering Vol. 02, No. 06, 2010, 2084-2091.

20. Jurafsky D. \& Martin J.H., "Speech and Language Processing" Parson Education, first Indian Reprint edition. 